Original Research

\title{
Radiological outcomes for endovascular treatment of posterior communicating artery aneurysms: a retrospective multicenter study of the occlusion rate
}

Alba Scerrati ${ }^{1,2, *}$ Cianluca Trevisi ${ }^{3}$ Carmelo Lucio Sturiale ${ }^{4}$ Francesco Salomi ${ }^{1}$ Pasquale De Bonis ${ }^{1,2}$ Andrea Saletti ${ }^{5}$ Annunziato Mangiola ${ }^{3,6}$, Alberto Tomatis ${ }^{3}$, Vincenzo Di Egidio ${ }^{7}$, Vera Vigo ${ }^{1,8}$, Alessandro Pedicelli ${ }^{9}$, lacopo Valente ${ }^{9}$, Oriela Rustemi ${ }^{10}$, Giacomo Beggio ${ }^{10}$, Giuseppe lannucci ${ }^{11}$, Luca Milonia ${ }^{12}$, Luca Ricciardi ${ }^{13}$, Amedeo Cervo ${ }^{12}$, Guglielmo Pero ${ }^{12}$, Mariangela Piano ${ }^{12}$

${ }^{1}$ Department of Neurosurgery, S. Anna University Hospital, 44121 Ferrara, Italy

${ }^{2}$ Department of Morphology, Surgery and Experimental Medicine, University of Ferrara, 44121 Ferrara, Italy

${ }^{3}$ Neurosurgical Unit, Santo Spirito Hospital, 65121 Pescara, Italy

${ }^{4}$ Department of Neurosurgery, Fondazione Policlinico Universitario A. Gemelli IRCCS, 00185 Rome, Italy

${ }^{5}$ Department of Interventional Neuroradiology, S. Anna University Hospital, 44121 Ferrara, Italy

${ }^{6}$ Department of Neurosciences, Imaging and Clinical Sciences, "C. D'Annunzio" University, 66100 Chieti, Italy

${ }^{7}$ Radiology Unit, Santo Spirito Hospital, 90044 Pescara, Italy

${ }^{8}$ The Stanford Neurosurgical Training and Innovation Center, Department of Neurosurgery, Stanford University, 94305 Palo Alto, CA, USA

${ }^{9}$ Department of Radiology, Fondazione Policlinico Universitario A. Cemelli IRCCS, 00185 Rome, Italy

${ }^{10}$ Department of Neurosurgery, San Bortolo Hospital, 70300 Vicenza, Italy

${ }^{11}$ Department of Neuroradiology, San Bortolo Hospital, 70300 Vicenza, Italy

${ }^{12}$ Department of Neuroradiology, Niguarda Hospital, 20162 Milan, Italy

${ }^{13}$ Department of Neurosurgery, Azienda Ospedaliera Sant'Andrea, Dipartimento NESMOS, 00186 Rome, Italy

*Correspondence: scrlba@unife.it (Alba Scerrati)

DOI:10.31083/j.jin2004093

This is an open access article under the CC BY 4.0 license (https://creativecommons.org/licenses/by/4.0/).

Submitted: 8 October 2021 Revised: 16 November 2021 Accepted: 18 November 2021 Published: 30 December 2021

Although several innovations in techniques and implantable devices were reported over the last decades, a consensus on the best endovascular treatment for intracranial aneurysms originating from the posterior communicating artery is still missing. This work investigates radiological outcomes of different endovascular techniques for posterior communicating artery aneurysms treatment in a retrospective multi-centric cohort. We included patients endovascularly treated for posterior communicating artery aneurysms from 2015 through 2020 in six tertiary referral hospitals. We evaluated the relationship between patients and aneurysms characteristics, baseline neurological status, radiological outcomes, and the different endovascular techniques. Overall, 250 patients were included in this study. Simple coiling was the most frequent treatment in 171 patients (68\%), followed by flow-diverter stenting in 32 cases (13\%). Complete occlusion was reported in 163 patients (65\%), near-complete occlusion in $43(17 \%)$, and incomplete occlusion in $44(18 \%)$. Radiological follow-up was available for 247 (98\%) patients. The occlusion rate was stable in $149(60 \%)$, improved in $49(19 \%)$, and worsened in 51 (21\%). No significant difference in exclusion rate was seen between ruptured and unruptured aneurysms at the last follow-up $(p=0.4)$. Posterior communicating artery thrombosis was reported in 25 patients $(9 \%)$, transient ischemic attack in $6(2 \%)$, and in 38 patients (15\%), subsequent procedures were needed due to incom- plete occlusion or reperfusion. Endovascular strategies for posterior communicating artery aneurysms represent effective and relatively safe treatments. Simple coiling provides a higher immediate occlusion rate, although recanalization has been frequently reported, conversely, flow-diversion devices provide good long-term radiological outcomes.

Keywords

Posterior communicating artery aneurysms; PcomA; Fetal posterior communicating artery; Fetal variant; Endovascular; Coiling; Flow-diverters; Intrasaccular devices

\section{Introduction}

Posterior communicating artery (PComA) is about $25 \%$ of all intracranial aneurysms and $50 \%$ of internal carotid artery (ICA) aneurysms, thus representing one of the most common vascular malformations encountered by neurosurgeons and neurointerventional radiologists [1]. Several anatomical variants of PComA aneurysms may have technical implications for their treatment, especially size, PComA fetal variant (4-29\% of cases) [2], and relationship of the PComA origin and aneurysm neck [3]. 
Surgical or hybrid treatments of PComA aneurysms are quite common [3-11], but few studies focus on the long-term outcome of large cohorts undergoing endovascular treatment. The recent advent of flow diverters and other newly designed endovascular devices has improved the feasibility and outcomes of PComA aneurysms. However, their longterm efficacy remains debated in particular cases, such as when associated with fetal-type PComA [12-17].

We retrospectively collected a large multicenter series of patients treated for PComA aneurysms with different endovascular techniques. Data about angioarchitecture features and related radiological outcomes and complications were analyzed according to the different endovascular techniques adopted. Indeed, this paper is mainly focused on the pure radiological technical aspect.

\section{Materials and methods}

\subsection{Study design}

This study is a retrospective multicenter analysis retrieving data of endovascular treatment of six different tertiary referral centers for cerebrovascular diseases in a timespan between January 2015 through December 2020.

\subsection{Inclusion and exclusion criteria}

We included patients $>18$ year-old with complete radiological and clinical reports and close follow-up. We excluded patients secondarily treated with endovascular devices after the failure of a previous clipping. We report on the pure radiological technical aspects of the procedures, not taking into account relevant clinical data. Patients gave written informed consent to use their medical and radiological data. The Institutional Review Board (AOSPFe EC) approved this study [EC 1254/M/2017].

\subsection{Data collection}

We collected for each patient: (1) demographics: age, gender; (2) neurological status at admission; (3) complications; (4) radiological outcome measured as presence or absence of subarachnoid hemorrhage (at CT-scan); aneurysm size and morphology; neck width; fetal-type PComA; treatment type; occlusion rate at post-operative imaging and follow-up (612-24 months); need for second re-treatments. In particular, we considered fetal-type a PComA/P1 ratio $>1$ with hemodynamic prevalence in the posterior cerebral artery from the anterior circulation (meaning the posterior cerebral artery territory filled from internal carotid artery injections) [18]. Angiographic features were evaluated by cerebral and rotational angiography with three-dimensional (3D) image reconstruction.

\subsection{Endovascular procedures}

The treatment strategy was based on a multidisciplinary consensus between vascular neurosurgeons and interventional neuroradiologists in all included patients. All endovascular procedures were performed under general anesthesia and via a femoral artery approach. Simple coiling, stentassisted coiling, flow-diversion, flow-diversion plus coiling and intravascular devices deployment procedures were performed according to single-center devices availability and protocols. Antiplatelets/anticoagulant drugs administered during the procedure and pre-and post-operatively were managed according to single-center protocols.

\subsection{Radiological follow-up}

MR angiography (MRa) or digital subtraction angiography (DSA) were performed at single or multiple follow-up times: 6, 12 and 24 months. The aneurysm occlusion rate was defined according to the Raymond-Roy (RR) classification incomplete occlusion (class I), residual neck (class II), and incomplete occlusion or residual aneurysm (class III) [19].

\subsection{Statistical analysis}

Kruskal-Wallis one-way analysis of variance and ChiSquared Test were used to assess differences among different endovascular treatments with respect to aneurysms' characteristics and treatment outcomes. To detect all pairs of a group that show significant differences, significant associations at the above omnibus tests were further analyzed through Dunn's posthoc comparisons and multinomial Generalized Linear Models with posthoc Bonferroni's correction, respectively. Contingency tables were also used to evaluate the association between radiological outcomes and aneurysms morphology and treatment. Independent samples Student's $T$-Test and Chi-Squared Test were used to analyze the differences in continuous and categorical variables among fetal and non-fetal PComA type. The significance level was set at 0.05. Statistical analysis was performed using JASP 0.13.1.0 - Chocolatey.org.

\section{Results}

\subsection{Demographics and aneurysms angio-architectural features}

Demographics and aneurysms' characteristics are summarized in Table 1. After institutional databases screening, 250 patients treated for PComA aneurysms with different endovascular techniques were included. Mean age was $60 \pm$ 14 years (range: 22-92 years), 217 were female (87\%), and 140 (56\%) of the included patients presented with SAH due to PComA aneurysm rupture at the time of hospitalization.

The great majority of aneurysms were saccular (235/250; 94\%) with a mean dome and neck size of $9 \mathrm{~mm}$ and $4 \mathrm{~mm}$, respectively. However, ruptured aneurysms had significantly smaller maximum size. On average, ruptured aneurysms had a significantly smaller maximum diameter and a narrower neck than unruptured ones.

\subsection{Type of treatment}

Simple coiling was performed in 171 patients (68\%), whereas flow-diverter stenting was performed in 32 (13\%). Other treatments, such as aneurysm remodeling, intravascular devices placement, stent-assisted coiling, and combination of coiling and flow-diverter stenting, were overall performed in 47 patients (19\%) (Tables 2,3).

The choice of the treatment modality significantly differed according to aneurysm presentation (ruptured/unruptured) 
Table 1. Demographics and aneurysms' characteristics split by aneurysm presentation (unruptured/ruptured).

\begin{tabular}{|c|c|c|c|c|c|}
\hline \multirow{2}{*}{\multicolumn{2}{|c|}{ Characteristics }} & \multicolumn{2}{|c|}{ Aneurysm presentation } & \multirow{2}{*}{$\begin{array}{c}\text { Entire population } \\
\qquad(n=250)\end{array}$} & \multirow{2}{*}{$p$} \\
\hline & & Unruptured $(n=110)$ & Ruptured $(n=140)$ & & \\
\hline \multicolumn{2}{|l|}{ Female gender } & $94(85)$ & $123(88)$ & $217(87)$ & 0.6 \\
\hline \multicolumn{2}{|l|}{ mean age in years } & $59 \pm 12$ & $61 \pm 15$ & $60 \pm 14$ & 0.1 \\
\hline \multicolumn{2}{|c|}{ Mean aneurysm maximum size in mm } & $11 \pm 5.8$ & $7.6 \pm 4$ & $9 \pm 5$ & $<0.001^{*}$ \\
\hline \multicolumn{2}{|c|}{ Mean aneurysm neck width in mm } & $4.3 \pm 2.3$ & $3.3 \pm 1.6$ & $3.7 \pm 2$ & $<0.001^{*}$ \\
\hline \multicolumn{2}{|c|}{ Mean aspect ratio (Size/Neck) } & $2.9 \pm 1.4$ & $2.5 \pm 1$ & $2.6 \pm 1.2$ & $0.01^{*}$ \\
\hline \multirow{5}{*}{ Aneurysm size } & $\leq 5 \mathrm{~mm}$ & $13(12)$ & $48(34)$ & $61(24)$ & \multirow{5}{*}{$<0.001^{*}$} \\
\hline & $6-10 \mathrm{~mm}$ & $51(46)$ & $65(46)$ & $116(46)$ & \\
\hline & $11-15 \mathrm{~mm}$ & $28(25)$ & $20(14)$ & $48(19)$ & \\
\hline & $16-20 \mathrm{~mm}$ & $12(11)$ & $5(4)$ & $17(7)$ & \\
\hline & $\geq 20 \mathrm{~mm}$ & $6(5)$ & $2(1)$ & $8(4)$ & \\
\hline \multirow{3}{*}{ Aneurysm morphology } & Saccular & $106(96)$ & $129(92)$ & $235(94)$ & \multirow{3}{*}{0.1} \\
\hline & Fusiform & $3(3)$ & $3(2)$ & $6(2)$ & \\
\hline & Blister & $1(1)$ & $8(6)$ & $9(4)$ & \\
\hline \multirow{2}{*}{ Fetal PComA } & Yes & $86(78)$ & $105(75)$ & $59(24)$ & \multirow{2}{*}{0.6} \\
\hline & No & $24(22)$ & $35(25)$ & $191(76)$ & \\
\hline
\end{tabular}

Legend: * Statistically Significant. Values are expressed as mean \pm standard deviation or number of patients (\%).

and aneurysm angioarchitecture features (Table 2). In particular, among the 110 patients with unruptured aneurysms, coiling was performed in 54 (49\%), flow diversion in 28 (25\%), balloon remodeling in 10 (10\%), and other treatments in 18 (16\%) (Tables 2,3). Among the 140 patients with ruptured aneurysms, coiling was performed in 117 (83\%), remodeling in $14(10 \%)$, flow diversion in $4(3 \%)$, and other treatments in 5 (3\%) (Table 2).

Moreover, coiling was preferred in the majority of ruptured aneurysms (84\%) and, regardless of the ruptured status, in the case of aneurysms of small size (mean $8.4 \mathrm{~mm}$ ) and narrow neck (mean $3.4 \mathrm{~mm}$ ). Contrariwise, the use of flow-diverters was mainly preferred in patients harboring unruptured aneurysms or, in general, aneurysms of larger size (mean $12.9 \mathrm{~mm}$ ) and wider neck (mean $5 \mathrm{~mm}$ ) (Tables 2,3,4). Tables 4 and 5 show significant differences between the two treatment types at the posthoc analysis of those quantitative or qualitative variables, which showed a significant difference at omnibus tests.

\subsection{Radiological outcome - entire population}

At immediate post-procedural imaging (MRa and/or DSA), a complete occlusion was reported in 163 patients (65\%), a near-complete occlusion in 43 (17\%), and an incomplete occlusion in 44 (18\%) (Table 2). A 6-months radiological follow-up was available in 245 (98\%) patients (5 patients lost at follow up) showing complete occlusion in 148 (61\%), near-complete occlusion in 74 (30\%), and incomplete in 23 (9\%) (Table 2).

A delayed improvement of occlusion rate in those patients with non-complete occlusion at post-procedural imaging (87 cases) was observed in 40 cases. In contrast, aneurysm recanalization during follow-up was observed in 51 patients (Tables 2,3).
A long-term radiological follow-up (24 months) was available in 105 patients (145 patients lost at follow-up). It showed complete occlusion in 64 (61\%) cases, near-complete occlusion in 32 (30\%) and incomplete in 9 (9\%) (Table 2). However, at the last follow-up, the occlusion rate was stable in 149 (60\%) of cases, improved in 49 (19\%) and worsened in 51 (21\%) (Table 3). The mean time of follow-up was 11 months, while the median follow-up time was 12 months.

\subsection{Radiological outcome-ruptured vs. unruptured presentation}

Results according to aneurysm presentation are summarized in Table 2 .

Complete post-procedural occlusion was obtained in 103 (74\%) ruptured and 60 (55\%) unruptured aneurysms. At early post-procedural control, near-complete exclusion was seen in 30 (21\%) ruptured and 13 (12\%) unruptured aneurysms, while an incomplete exclusion in 7 (5\%) ruptured and 37 (34\%) unruptured, respectively. This difference appeared significant $(p<0.001)$.

However, 33 (30\%) unruptured aneurysms showed an occlusion rate improvement over time, showing a complete occlusion at last follow-up in 69 (63\%) cases, and a nearcomplete exclusion in 31 (28\%) patients. On the other hand, a delayed improvement was less frequent in ruptured aneurysms, which showed a complete occlusion rate of $71 \%$ and a near-complete exclusion rate of $24 \%$ at the last followup. Therefore, no significant difference in exclusion rate was seen between ruptured and unruptured aneurysms at the last follow-up ( $p=0.4$ ). Recanalization during follow-up was documented in 34 (24\%) ruptured and 17 (16\%) unruptured aneurysms, respectively. However, this difference was not significant $(p=0.1)$.

A subgroup analysis comparing ruptured and unruptured aneurysms showed that $47 / 54$ patients (87\%) with unrup- 
Table 2. Treatments, radiological outcomes and complications split by aneurysm presentation (unruptured/ruptured).

\begin{tabular}{|c|c|c|c|c|c|}
\hline \multirow{2}{*}{ Characteristics } & & \multicolumn{2}{|c|}{ Aneurysm presentation } & \multirow{2}{*}{$\begin{array}{c}\text { Entire population } \\
\qquad(n=250)\end{array}$} & \multirow{2}{*}{$p$} \\
\hline & & Unruptured $(n=110)$ & Ruptured $(n=140)$ & & \\
\hline \multirow{6}{*}{ Endovascular treatment } & Simple coiling & $54(49)$ & $117(83)$ & $171(68)$ & \multirow{6}{*}{$<0.001^{*}$} \\
\hline & Remodeling & $10(10)$ & $14(10)$ & $24(10)$ & \\
\hline & Stent assisted coiling & $4(4)$ & $1(1)$ & $5(2)$ & \\
\hline & Flow diverter & $28(25)$ & $4(3)$ & $32(13)$ & \\
\hline & Simple coiling + flow diverter & $7(6)$ & $1(1)$ & $8(3)$ & \\
\hline & Intrasaccular device & $7(6)$ & $3(2)$ & $10(4)$ & \\
\hline \multirow{3}{*}{ Post-treatment occlusion rate } & Complete & $60(55)$ & $103(74)$ & $163(65)$ & \multirow{3}{*}{$<0.001$} \\
\hline & Near complete & $13(12)$ & $30(21)$ & $43(17)$ & \\
\hline & Incomplete & $37(33)$ & $7(5)$ & $44(18)$ & \\
\hline \multirow{4}{*}{ Occlusion rate at 6 months } & $N^{\circ}$ Patients & $\mathrm{n}=105$ & $\mathrm{n}=140$ & $\mathrm{n}=245$ & \multirow{4}{*}{0.2} \\
\hline & Complete & $61(58)$ & $87(62)$ & $148(61)$ & \\
\hline & Near complete & $30(29)$ & $44(32)$ & $74(30)$ & \\
\hline & Incomplete & $14(13)$ & $9(6)$ & $23(9)$ & \\
\hline \multirow{4}{*}{ Occlusion rate at 12 months } & $N$ Patients & $\mathrm{n}=97$ & $\mathrm{n}=103$ & $\mathrm{n}=200$ & \multirow{4}{*}{0.8} \\
\hline & Complete & $58(60)$ & $63(61)$ & $121(60)$ & \\
\hline & Near complete & $28(29)$ & $31(30)$ & $59(30)$ & \\
\hline & Incomplete & $11(11)$ & $9(9)$ & $20(10)$ & \\
\hline \multirow{4}{*}{ Occlusion rate at 24 months } & $N$ Patients & $\mathrm{n}=54$ & $\mathrm{n}=51$ & $\mathrm{n}=105$ & \multirow{4}{*}{0.8} \\
\hline & Complete & $34(63)$ & $30(59)$ & $64(61)$ & \\
\hline & Near complete & $16(30)$ & $16(31)$ & $32(30)$ & \\
\hline & Incomplete & $4(7)$ & $5(10)$ & $9(9)$ & \\
\hline \multirow{6}{*}{ Type of re-treatment } & $N^{\circ}$ Procedures & $\mathrm{n}=12$ & $\mathrm{n}=29$ & $\mathrm{n}=41$ & \multirow{6}{*}{0.2} \\
\hline & Simple coiling & $2(17)$ & $4(14)$ & $6(15)$ & \\
\hline & Remodeling & 0 & $2(7)$ & $2(5)$ & \\
\hline & Stent assisted coiling & $2(17)$ & $10(34)$ & $12(29)$ & \\
\hline & Flow diverter & $5(42)$ & $12(41)$ & $17(41)$ & \\
\hline & Surgery & $3(25)$ & $1(3)$ & $4(10)$ & \\
\hline \multirow{5}{*}{ Treatment related complications } & No complications & $100(90)$ & $116(83)$ & $216(87)$ & \multirow{5}{*}{0.1} \\
\hline & Hemorrhage & $1(1)$ & $1(0.7)$ & $2(0.8)$ & \\
\hline & Thrombosis & $5(5)$ & $18(13)$ & $23(9)$ & \\
\hline & TIA/Stroke & $4(4)$ & $2(1.3)$ & $6(2)$ & \\
\hline & Death Unrelated to Treatment & 0 & $3(2)$ & $3(1.2)$ & \\
\hline \multicolumn{2}{|l|}{ Recanalization during follow-up } & $17(16)$ & $34(24)$ & $51(21)$ & 0.1 \\
\hline
\end{tabular}

Legend: * Statistically Significant. Values are expressed as mean \pm standard deviation or number of patients (\%).

tured PComA aneurysms undergoing coiling showed a complete occlusion at post-procedural imaging, compared with 88/117 (75\%) in the ruptured group. A near-complete occlusion after coiling was obtained in unruptured 7 (13\%) and 27 (23\%) ruptured aneurysms, respectively. An incomplete occlusion was observed only in 2 cases treated by simple coiling of the ruptured group. Nonetheless, these differences in occlusion rate were not statistically significant $(p=0.1)$. The frequency of recanalization after simple coiling was similar between the unruptured and ruptured groups, with 14 (26\%) unruptured and 32 (27\%) ruptured aneurysms recanalized during follow-up.

\subsection{Complications and re-treatments}

Overall, 3 patients with ruptured aneurysms died in the early post-procedural period for complications not related to endovascular treatment ( 2 sudden cardiac deaths and 1 res- piratory failure). Intraprocedural PComA thrombosis was the most frequent complication reported in 23 patients (9\%). All of them were immediately pharmacologically treated, obtaining recanalization. Transient ischemic attacks (TIA) or strokes with no evidence of intraoperative PComA thrombosis were instead reported in 6 cases (2\%). Two cases of intraprocedural aneurysm rupture during coiling occurred, although no major neurological sequelae were registered.

Thirty-eight aneurysms (15\%) with incomplete occlusion or recanalization underwent 41 secondary procedures as $3 \mathrm{pa}-$ tients needed 2 re-treatments. In particular, a flow-diverter stent was positioned in 17 (41\%) cases, a stent-assisted recoiling in 12 (29\%), and a simple recoiling in 6 (14\%). Microsurgical clipping and aneurysm remodeling were preferred in 4 (10\%) and 2 (5\%) of recanalization, respectively (Tables 2,3). 
Table 3. Results according to initial endovascular treatment.

\begin{tabular}{|c|c|c|c|c|c|c|c|c|c|}
\hline & & \multicolumn{6}{|c|}{ Initial endovascular treatment } & \multirow[b]{2}{*}{ Total } & \multirow{3}{*}{$p$} \\
\hline & & Simple Coiling & Remodeling & Stent-assisted Coiling & Flow Diverter & $\begin{array}{l}\text { Simple Coiling + } \\
\text { Flow Diverter }\end{array}$ & Intrasaccular Device & & \\
\hline & & $(n=171)$ & $(n=24)$ & $(n=5)$ & $(n=32)$ & $(n=8)$ & $(n=10)$ & $(n=250)$ & \\
\hline Mean Age in years & & $60 \pm 14$ & $60 \pm 13$ & $53 \pm 9$ & $63 \pm 14$ & $53 \pm 15$ & $57 \pm 9$ & $60 \pm 14$ & 0.3 \\
\hline Mean aneurysm maximum size in $\mathrm{mm}$ & & $8.4 \pm 4.7$ & $7.9 \pm 3.7$ & $7.5 \pm 2.6$ & $12.9 \pm 7.3$ & $12.6 \pm 5.2$ & $8.4 \pm 2.4$ & $9 \pm 5$ & $0.003^{*}$ \\
\hline Mean aneurysm neck width in mm & & $3.4 \pm 1.7$ & $3.8 \pm 2.3$ & $3.4 \pm 0.7$ & $5 \pm 2.6$ & $5.7 \pm 2.4$ & $3.6 \pm 0.7$ & $3.7 \pm 2$ & $<0.001^{*}$ \\
\hline Mean aspect ratio (Size/Neck) & & $2.7 \pm 1.2$ & $2 \pm 0.5$ & $2.2 \pm 0.6$ & $2.7 \pm 1.4$ & $2.5 \pm 1.2$ & $2.5 \pm 0.8$ & $2.6 \pm 1.2$ & 0.1 \\
\hline Aneurysm presentation & Ruptured & $117(68)$ & $14(58)$ & $1(20)$ & $4(12)$ & $1(12)$ & $3(30)$ & $140(56)$ & $<0.001^{*}$ \\
\hline \multirow{3}{*}{ Aneurysm morphology } & Saccular & $161(94)$ & $23(96)$ & $5(100)$ & $30(94)$ & $6(75)$ & $10(100)$ & $235(94)$ & \multirow{3}{*}{$0.02^{*}$} \\
\hline & Fusiform & $2(1)$ & $1(4)$ & 0 & $1(3)$ & $2(25)$ & 0 & $6(2)$ & \\
\hline & Blister & $8(5)$ & 0 & 0 & $1(3)$ & 0 & 0 & $9(4)$ & \\
\hline \multirow{4}{*}{ Aneurysm maximum size } & $\leq 5 \mathrm{~mm}$ & $47(28)$ & $7(29)$ & $1(20)$ & $5(16)$ & 0 & $1(10)$ & $61(24)$ & \multirow{4}{*}{0.004} \\
\hline & $6-10 \mathrm{~mm}$ & $82(48)$ & $12(50)$ & $3(60)$ & $9(28)$ & $4(50)$ & $6(60)$ & $116(46)$ & \\
\hline & $11-15 \mathrm{~mm}$ & $31(18)$ & $4(17)$ & $1(20)$ & $8(25)$ & $1(12)$ & $3(30)$ & $48(19)$ & \\
\hline & $16-20 \mathrm{~mm}$ & $7(4)$ & $1(4)$ & 0 & $6(19)$ & $3(38)$ & 0 & $17(7)$ & \\
\hline \multirow{4}{*}{ Treatment related complications } & None & $148(87)$ & $20(83)$ & $4(80)$ & $29(91)$ & $8(100)$ & $10(100)$ & $219(88)$ & \multirow{4}{*}{0.1} \\
\hline & Hemorrhage & $2(1)$ & 0 & 0 & 0 & 0 & 0 & $2(1)$ & \\
\hline & Thrombosis & $19(11)$ & $4(17)$ & 0 & $0(6)$ & 0 & 0 & $23(9)$ & \\
\hline & TIA/Stroke & $2(1)$ & 0 & $1(20)$ & $3(9)$ & 0 & 0 & $6(2)$ & \\
\hline \multirow{3}{*}{ Post-treatment occlusion rate } & Complete & $135(79)$ & $16(67)$ & $4(80)$ & $4(12)$ & $3(38)$ & $1(10)$ & $163(65)$ & \multirow{3}{*}{$<0.001^{*}$} \\
\hline & Near Complete & $34(20)$ & $7(29)$ & 0 & $1(3)$ & $1(12)$ & 0 & $43(17)$ & \\
\hline & Incomplete & $2(1)$ & $1(4)$ & $1(20)$ & $27(85)$ & $4(50)$ & $9(90)$ & $44(18)$ & \\
\hline \multirow{3}{*}{ Occlusion rate at last follow-up } & Complete & $116(68)$ & $19(79)$ & $4(40)$ & $17(53)$ & $5(63)$ & $7(70)$ & $168(67)$ & \multirow{3}{*}{0.1} \\
\hline & Near Complete & $45(26)$ & $5(21)$ & $1(20)$ & $8(25)$ & $2(25)$ & $3(30)$ & $64(26)$ & \\
\hline & Incomplete & $10(6)$ & 0 & 0 & $7(22)$ & $1(12)$ & 0 & $18(7)$ & \\
\hline \multirow{4}{*}{$\begin{array}{l}\text { Delayed occlusion in non-complete } \\
\text { early occlusion }\end{array}$} & Number of patients & $n=30$ & $n=7$ & $n=1$ & $n=28$ & $n=5$ & $n=9$ & $n=80$ & \multirow{4}{*}{0.06} \\
\hline & Complete & $11(37)$ & $4(57)$ & $1(100)$ & $14(50)$ & $3(60)$ & $6(78)$ & $39(49)$ & \\
\hline & Near Complete & $1(3)$ & 0 & 0 & $6(21)$ & $1(20)$ & $3(22)$ & $11(13.7)$ & \\
\hline & Incomplete & $18(60)$ & $3(43)$ & 0 & $8(29)$ & $1(20)$ & 0 & $30(37.5)$ & \\
\hline \multirow[b]{2}{*}{ Recanalization during follow-up } & Number of patients & $n=171$ & $n=24$ & $n=4$ & $n=30$ & $n=8$ & $n=9$ & $n=246$ & \multirow[b]{2}{*}{$0.01^{*}$} \\
\hline & Yes & $46(27)$ & $3(12.5)$ & $1(25)$ & 0 & 0 & 0 & $51(21)$ & \\
\hline \multirow{3}{*}{$\begin{array}{l}\text { Overall result at follow-up compared } \\
\text { to early control }\end{array}$} & Improved & $12(7)$ & $4(17)$ & 0 & $20(62.5)$ & $4(50)$ & $9(90)$ & $49(19)$ & \multirow{3}{*}{$<0.001^{*}$} \\
\hline & Stable & $113(66)$ & $17(71)$ & $3(75)$ & $12(37.5)$ & $4(50)$ & 0 & $149(60)$ & \\
\hline & Worsened & $46(27)$ & $3(12)$ & $1(25)$ & 0 & 0 & $1(10)$ & $51(21)$ & \\
\hline
\end{tabular}


Table 3. Continued.

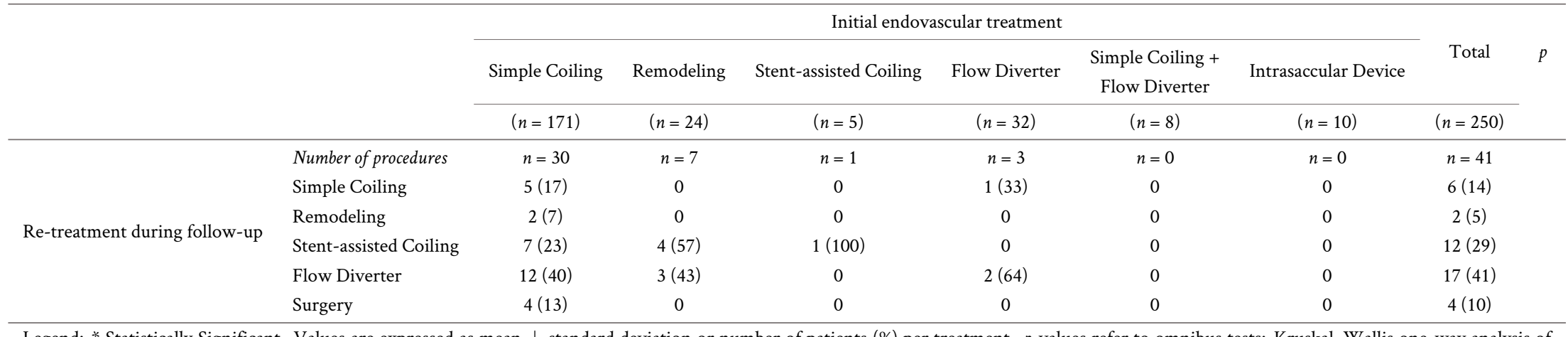

Legend: * Statistically Significant. Values are expressed as mean \pm standard deviation or number of patients (\%) per treatment. $p$-values refer to omnibus tests: Kruskal-Wallis one-way analysis of variance (quantitative variables) and Chi-Squared Test (qualitative variables).

Table 4. Quantitative variables - significant differences among endovascular treatments.

\begin{tabular}{llc}
\hline \multicolumn{2}{c}{ Table 4. Quantitative variables - significant differences among endovascular treatments. } \\
\hline Variable & Treatments comparison & $p$ \\
\hline & Simple Coiling - Flow Diverter & $<0.001$ \\
& Simple Coiling - Simple Coiling + Flow Diverter & 0.007 \\
& Remodeling - Flow-Diverter & 0.003 \\
Mean aneurysm maximum size & Remodeling - Simple Coiling + Flow Diverter & 0.01 \\
& Stent Assisted Coiling - Flow Diverter & 0.04 \\
& Stent Assisted Coiling - Simple Coiling + Flow Diverter & 0.04 \\
\hline \multirow{5}{*}{ Mean aneurysm neck width } & Simple Coiling - Flow Diverter & $<0.001$ \\
& Simple Coiling - Simple Coiling + Flow Diverter & $<0.001$ \\
& Remodeling - Flow-Diverter & 0.01 \\
& Remodeling - Simple Coiling + Flow Diverter & 0.01
\end{tabular}

Legend: Post-Hoc test of significant quantitative variables at Kruskal-Wallis one-way analysis of variance. Pairwise comparison of significant differences. Variables' values and Kruskal-Wallis test's significance are reported in Table 3. 


\subsection{Radiological outcome predictors}

A complete occlusion at first treatment was significantly more common in small $(p<0.001)$ and ruptured $(p<0.001)$ aneurysms. A 6-months complete occlusion was mostly observed in patients who underwent coiling (135/163; 79\%) compared with other endovascular treatments ( 28 cases, $80 \%$ ) $(p<0.001)$ (Tables 2,5). At long-time radiological followup instead, on the one hand, we observed a significant improvement of the occlusion rate $(20 / 49$ out of cases) in patients treated with flow-diverter stents $(p<0.001)$, while on the other a significant rate of recanalization (considered as an increase in contrast filling of the aneurysm once it was completely occluded) $(46 / 51$ out of cases) in those treated with coiling $(p<0.001)$ (Tables 3,5$)$.

Finally, no significant difference in occlusion rate was observed at the last follow-up according to the different endovascular treatments (Tables 3,5).

\subsection{Fetal-type PComA}

Comparison between fetal and non-fetal type PComA is reported in Table 6.

A Fetal PComA type was found in 59 patients (24\%). Simple coiling was performed in 43 cases (73\%), flow-diversion in $6(10 \%)$, remodeling in $5(9 \%)$ and other treatments in 5 (9\%). This subgroup of patients showed 7 blister-like aneurysms, differently from the non-fetal variant (2 cases) (Table 6). This difference was statistically significant $(p<$ 0.001 ). However, no statistically significant differences were seen in demographics, mean aneurysms size, clinical presentation, type of endovascular treatments, early and delayed occlusion rate, and frequency of recanalization between the two PComA type groups.

\section{Discussion \\ 4.1 General considerations}

Our paper mainly focuses on the pure radiological technical aspect of the procedures, lacking relevant clinical data. Our results seem to suggest that endovascular Treatment for PComA aneurysms is safe and effective. Proximal location, clear angiographic anatomy and comfortable catheter access represent favorable elements when considering embolization. Conversely, whenever the PComA has to be sacrificed, the fetal variant has been reported a higher risk for ischemic complications [20-22].

To the best of our knowledge, the present work represents the largest multicenter case series investigating radiological outcomes after endovascular treatments for PComA aneurysms at long follow-up ( $>24$ months).

In this series, endovascular treatments showed an occlusion rate (complete and near-complete occlusion) as high as $65 \%$ on post-procedural imaging and improved to $90-93 \%$ in long-term follow-up evaluations (Tables 1,2,3). When comparing the different techniques, there were no significant differences in aneurysm occlusion nor a higher rate of hemorrhagic and thromboembolic complications at the last imaging follow-up. However, we should consider that the different groups of treatment are heterogeneous in terms of aneurysms size or neck width (larger for FD or coiling + FD) and aneurysm presentation (more frequently ruptured in the coiling group) Table 3.

Raymond et al. [23], in their series of 43 patients suffering from PComA aneurysms and treated endovascularly, reported a recurrence rate of $37.2 \%$. In addition, in the ISAT trial, coiled aneurysms in the PComA location reported a significantly higher risk of recurrence requiring re-treatment [24].

The difference between our results showing a lower recurrence rate (15\%) and the studies above could be explained by considering we included aneurysms treated with different techniques. In contrast, patients were mainly treated by coiling in Raymond [23] and the ISAT trial. Indeed, a possible explanation could be that a better patient-based selection of the devices was performed. More than $1 / 3$ of patients were treated differently than coiling alone.

In our series, coiling was the most common procedure (68\%), associated with an $18 \%$ recurrence rate. Indeed, recanalization (increase in contrast filling of the aneurysm once it was completely occluded) had a significant association with simple coiling $(p=0.01)$ and never occurred with flow diversion. This is not unexpected since aneurysms treated with flow diverters present a minimal risk of recanalization once occlusion is achieved [25]. Higher rates of recanalization with coiling are also reported by Choi et al. [18] in their series of 480 coiled PComA aneurysms, 33.1\% showed a recanalization during the follow-up.

In our series, recanalization (increase in contrast filling of the aneurysm once it was completely occluded) during follow-up was overall reported in $20 \%$ of patients. Retreatment was performed in $15 \%$. Flow-diversion was chosen for re-treatment in most of these cases (41\%). Even if most of the patients with a recanalization were retreated, in the choice of proposing re-treatment, we should consider the relatively low risk of rebleeding (varying from $0.6 \%$ to $2.6 \%$ [26]) and the immediate risks of morbidity and mortality associated with each procedure in the re-treatment session. Moreover, our data showed a tendency towards delayed (at 12 and 24 months) improvement of occlusion rates of patients with an incomplete early occlusion at 6 months followup. This could be explained considering patients undergoing flow-diversion usually present a "delayed" occlusion if compared to coiling (more frequently completely occluding the aneurysm at early controls $(p<0.001))$. Indeed, the occlusion rate significantly improved at final follow-up in patients treated using a flow diverter $(p<0.001)$. The complications rate was $12 \%$, most of which were PComA thrombosis. Indeed, all thrombosis was treated during the endovascular procedure and did not cause any post-procedural neurological deficit.

\subsection{Comparison between different types of procedure}

Simple coiling was the procedure of choice in nearly $70 \%$ of patients, preferred for small and ruptured aneurysms. The 
Table 5. Qualitative variables - significant differences among endovascular treatments.

\begin{tabular}{|c|c|c|}
\hline Treatment & $\begin{array}{l}\text { Comparison of the likelihood of qualitative variables } \\
\text { (increased likelihood vs. decreased likelihood) }\end{array}$ & PBonferroni \\
\hline \multirow{6}{*}{ Simple coiling } & Presentation (Ruptured vs. Unruptured) & $<0.001$ \\
\hline & Early Occlusion (Complete vs. Incomplete) & $<0.001$ \\
\hline & Early Occlusion (Near Complete vs. Incomplete) & $<0.001$ \\
\hline & Recanalization (yes vs. no) & $<0.001$ \\
\hline & Overall Result at Follow-Up Compared to Early Control (Stable vs. Improved) & $<0.001$ \\
\hline & Overall Result at Follow-Up Compared to Early Control (Worsened vs. Improved) & $<0.001$ \\
\hline Remodeling & Morphology (Saccular vs. Blister) & $<0.001$ \\
\hline \multirow{7}{*}{ Flow-diverter } & Presentation (Unruptured vs. Ruptured) & $<0.001$ \\
\hline & Early Occlusion (Incomplete vs. Complete) & $<0.001$ \\
\hline & Early Occlusion (Incomplete vs. Near Complete) & $<0.001$ \\
\hline & Recanalization (no vs. yes) & $<0.001$ \\
\hline & Overall Result at Follow-Up Compared to Early Control (Improved vs. Stable) & 0.001 \\
\hline & Overall Result at Follow-Up Compared to Early Control (Improved vs. Worsened) & $<0.001$ \\
\hline & Overall Result at Follow-Up Compared to Early Control (Stable vs. Worsened) & 0.008 \\
\hline \multirow{2}{*}{ Simple coiling + flow diverter } & Presentation (Unruptured vs. Ruptured) & 0.04 \\
\hline & Recanalization (no vs. yes) & 0.01 \\
\hline \multirow{6}{*}{ Intravascular device } & Morphology (Saccular vs. Blister) & 0.01 \\
\hline & Morphology (Saccular vs. Fusiform) & 0.01 \\
\hline & Early Occlusion (Incomplete vs. Complete) & 0.01 \\
\hline & Early Occlusion (Incomplete vs. Near Complete) & 0.01 \\
\hline & Overall Result at Follow-Up Compared to Early Control (Improved vs. Stable) & 0.01 \\
\hline & Overall Result at Follow-Up Compared to Early Control (Improved vs. Worsened) & 0.04 \\
\hline
\end{tabular}

Legend: Post-Hoc test of significant qualitative variables at Chi-Squared Test.

The table shows the significant difference of variables in each treatment group. In brackets the qualitative variable with increased

likelihood $v$ s. the variable with a decreased likelihood Variables' frequencies and Chi-Squared Test' significance are reported in Table 3.

early occlusion rate was $79 \%$, and the complication rate was $13 \%$, being thrombosis the most frequent. Coiled aneurysms tend to recanalize over time (Fig. 1), with a statistically significant difference $(p=0.01)$ compared to other treatments.

Stent-assisted coiling was not common as the first treatment (2\%) but was the treatment of choice in $29 \%$ of retreated cases. The use of this technique as the first line is probably limited, especially in wide-neck PComA aneurysms with a fetal PCA because these tend to incorporate the origin of the fetal PCA and the ICA [27].

Flow-diversion was performed in $13 \%$ of patients, and it was associated with coiling in 8 (3\%) patients (Fig. 2). Although it is usually preferred in unruptured aneurysms (because of the need for dual antiplatelet therapy), in our series 4 ruptured aneurysms were treated with flow-diverters. Indeed, the safety of dual antiplatelet administration in acute $\mathrm{SAH}$ is still controversial for the possible increased risk of bleeding. However, evidence exists supporting the safety of this therapy even in hemorrhagic settings $[28,29]$. Moreover, flow-diversion was the chosen procedure in more than $40 \%$ of re-treatments. The association of coiling and subsequently flow diversion could represent a good option to benefit from the immediate effect of occlusion of coils and the long-term effect of vessel reconstruction of flow diverters (Fig. 2). However, the drawback of this strategy is the need for long-term antiplatelet therapy compared to the coiling alone group.

Intravascular devices are increasingly emerging as endovascular treatment, especially for bifurcation aneurysms [30]. We reported a relatively large number (10 patients) of intravascular devices Woven EndoBridge (WEB)@ placement for PComA aneurysms if compared to the present knowledge [31-33]. In 3 cases, the aneurysms were ruptured. The radiological outcome for all the treated aneurysms (ruptured and unruptured) at long-term was complete/nearcomplete occlusion in $100 \%$ of patients, with no complications (Fig. 3).

This procedure could be a good alternative to simple coiling in ruptured aneurysms and patients with contraindications for antiplatelet therapy. However, since this technique is relatively new, the learning curve, the device's prompt availability, and its costs should be carefully considered.

Lastly, aneurysm remodeling, namely coiling with the assistance of a removable device (either a balloon or a Comaneci device in our series), was used in 24 (10\%) patients, with 7 (29\%) cases requiring a second treatment during follow-up. No significant differences in aneurysm characteristics, early and late occlusion compared to simple coiling were found. 
Table 6. Results according to posterior communicating artery type.

\begin{tabular}{|c|c|c|c|c|}
\hline & & \multicolumn{2}{|c|}{ Posterior communicating artery type } & \multirow{3}{*}{$p$} \\
\hline & & Non-Fetal & Fetal & \\
\hline & & $(n=191)$ & $(n=59)$ & \\
\hline \multicolumn{2}{|l|}{ Female gender } & $164(86)$ & $53(90)$ & 0.4 \\
\hline \multicolumn{2}{|l|}{ Mean age in years } & $60 \pm 14$ & $62 \pm 12$ & 0.4 \\
\hline \multicolumn{2}{|l|}{ Mean aneurysm maximum size in $\mathrm{mm}$} & $9.2 \pm 5.3$ & $8.6 \pm 4.9$ & 0.4 \\
\hline \multicolumn{2}{|l|}{ Mean aneurysm neck width in mm } & $3.6 \pm 2$ & $4.1 \pm 2.1$ & 0.2 \\
\hline \multicolumn{2}{|l|}{ Mean aspect ratio (Size/Neck) } & $2.8 \pm 1.3$ & $2.4 \pm 1$ & 0.1 \\
\hline \multirow{5}{*}{ Aneurysm maximum size } & $\leq 5 \mathrm{~mm}$ & $45(23.6)$ & $16(27.1)$ & \multirow{5}{*}{0.9} \\
\hline & $6-10 \mathrm{~mm}$ & $89(46.6)$ & $27(45.8)$ & \\
\hline & $11-5 \mathrm{~mm}$ & $36(18.8)$ & $12(20.3)$ & \\
\hline & $16-20 \mathrm{~mm}$ & $14(7.3)$ & $3(5.1)$ & \\
\hline & $>20 \mathrm{~mm}$ & $7(3.7)$ & $1(1.7)$ & \\
\hline \multirow{3}{*}{ Aneurysm morphology } & Saccular & $185(96)$ & $51(86)$ & \multirow{3}{*}{$<0.001^{*}$} \\
\hline & Fusiform & $5(3)$ & $1(2)$ & \\
\hline & Blister & $2(1)$ & $7(12)$ & \\
\hline \multirow{2}{*}{ Aneurysm presentation } & Unruptured & $86(45)$ & $24(41)$ & \multirow{2}{*}{0.6} \\
\hline & Ruptured & $105(55)$ & $35(59)$ & \\
\hline \multirow{6}{*}{ Endovascular treatment } & Simple Coiling & $128(67)$ & $43(72.9)$ & \multirow{6}{*}{0.5} \\
\hline & Remodeling & $19(9.9)$ & $5(8.5)$ & \\
\hline & Stent Assisted Coiling & $3(1.6)$ & $2(3.4)$ & \\
\hline & Flow Diverter & $26(13.6)$ & $6(10.2)$ & \\
\hline & Simple Coiling + Flow Diverter & $8(4.2)$ & 0 & \\
\hline & Intravascular Device & $7(3.7)$ & $3(4.9)$ & \\
\hline \multirow{4}{*}{ Treatment complications } & No Treatment Related Complications & $169(89)$ & $50(85)$ & \multirow{4}{*}{0.7} \\
\hline & Subarachnoid Hemorrhage & $2(1)$ & 0 & \\
\hline & Thrombosis & $16(8)$ & $7(12)$ & \\
\hline & TIA/Stroke & $4(2)$ & $2(3)$ & \\
\hline \multirow{3}{*}{ Early occlusion } & Complete & $128(67)$ & $35(59)$ & \multirow{3}{*}{0.2} \\
\hline & Near Complete & $28(15)$ & $15(26)$ & \\
\hline & Incomplete & $35(18)$ & $9(15)$ & \\
\hline \multirow{3}{*}{$\begin{array}{l}\text { Delayed occlusion in non-complete } \\
\text { early occlusion }\end{array}$} & Complete & $30(52.6)$ & $10(43.5)$ & \multirow{3}{*}{0.5} \\
\hline & Partial & $8(14)$ & $2(8.7)$ & \\
\hline & No Delayed Occlusion & $19(33.3)$ & $11(47.8)$ & \\
\hline \multicolumn{2}{|l|}{ Recanalization during follow-up } & $38(20)$ & $13(22)$ & 0.7 \\
\hline
\end{tabular}

\subsection{Fetal-type PComA aneurysms}

Fetal-type PComA was present in 59 patients, and aneurysms more frequently presented a blister morphology ( $12 \%$ vs. $1 \%$ of non-fetal PComA). These kinds of aneurysms can be considered a challenge for treatment because the origin of the fetal PComA arises from the aneurysm sidewall [34].

Our results did not show differences between fetal and non-fetal type PComA aneurysms in terms of the type of treatment, complications (higher rate of thrombosis, $12 \%$ vs. $8 \%$, but not statistically significant $(p=0.7))$, early and delayed occlusion and recanalization.

These data stand out when compared to the previous studies. Indeed, a higher rate of incomplete occlusion was reported when a fetal-type PComA was present, particularly with flow-diversion treatment [12, 13, 15, 35]. Possible explanations for the failure of flow diversion in fetal type PComA aneurysms are based on the fact that flow through the fetal PComA and the aneurysm sac remains plentiful for the high physiologic demand and does not allow the stasis needed for the thrombotic process and occlusion to occur [36].

Discrepancies in our results could be explained considering the previous series reported a smaller number of patients (between 4 and 7), and mean follow-up was between 5 and 9 months. Indeed, as we demonstrated, flow-diversion procedures increase the rate of occlusion at long-term follow-up. Moreover, Enriquez-Marulanda et al. [37], in their series of 35 patients, did not report the presence of fetal PComA associated with increased risk of incomplete occlusion; and Choi et al. [18] in their series of 480 coiled aneurysms reported re- 


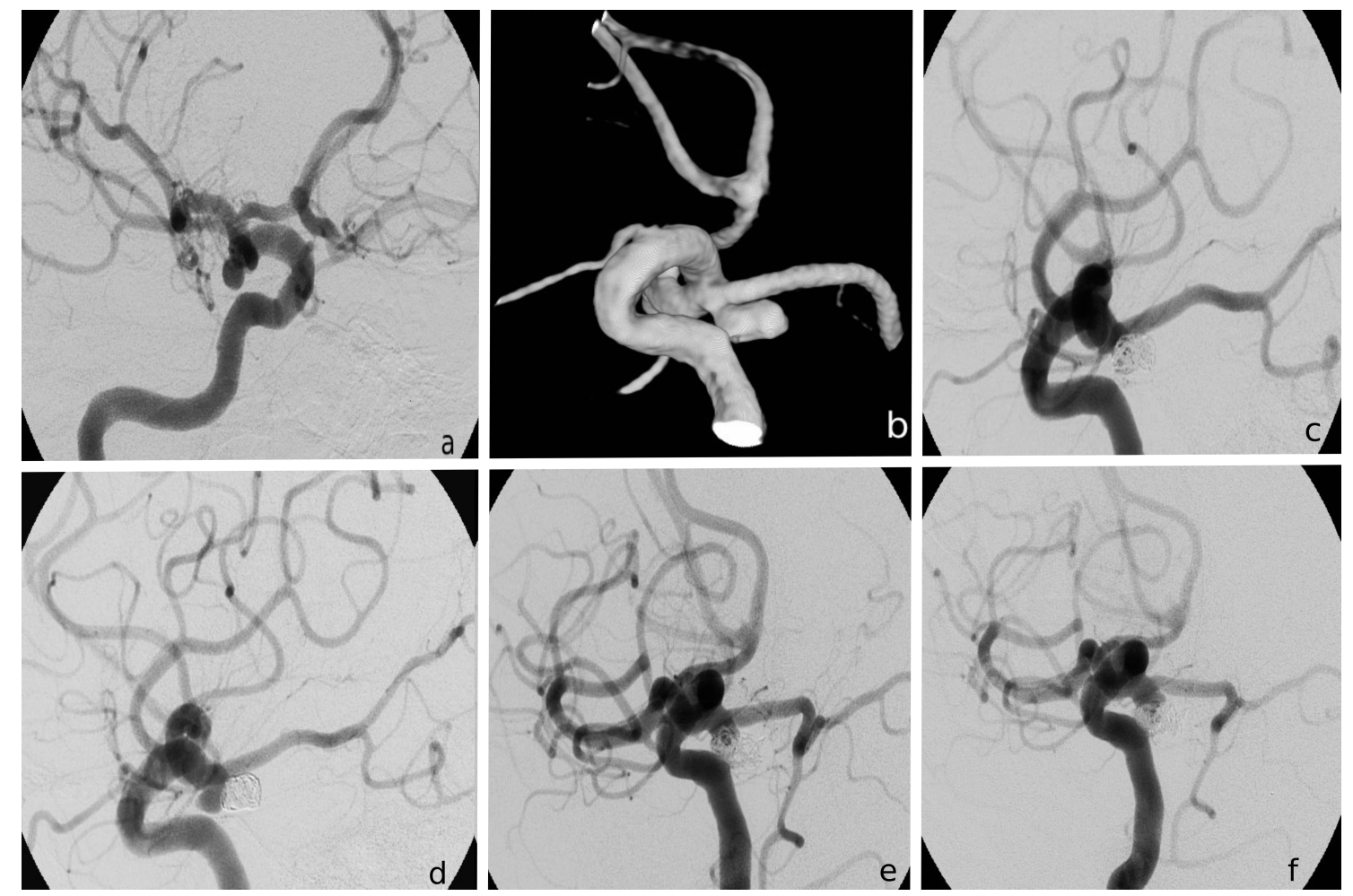

Fig. 1. DSA of coiling of a PComA aneurysm. 68 years old woman with an acute onset of III cranial nerve palsy and evidence of PComA aneurysm at DSA $(\mathrm{a}, \mathrm{b})$. Coiling procedure with complete occlusion of the aneurysm (c). Six months follow-up DSA showing recanalization (d). Re-treatment with coiling (e) and 6 months follow-up DSA showing a small residual (f).
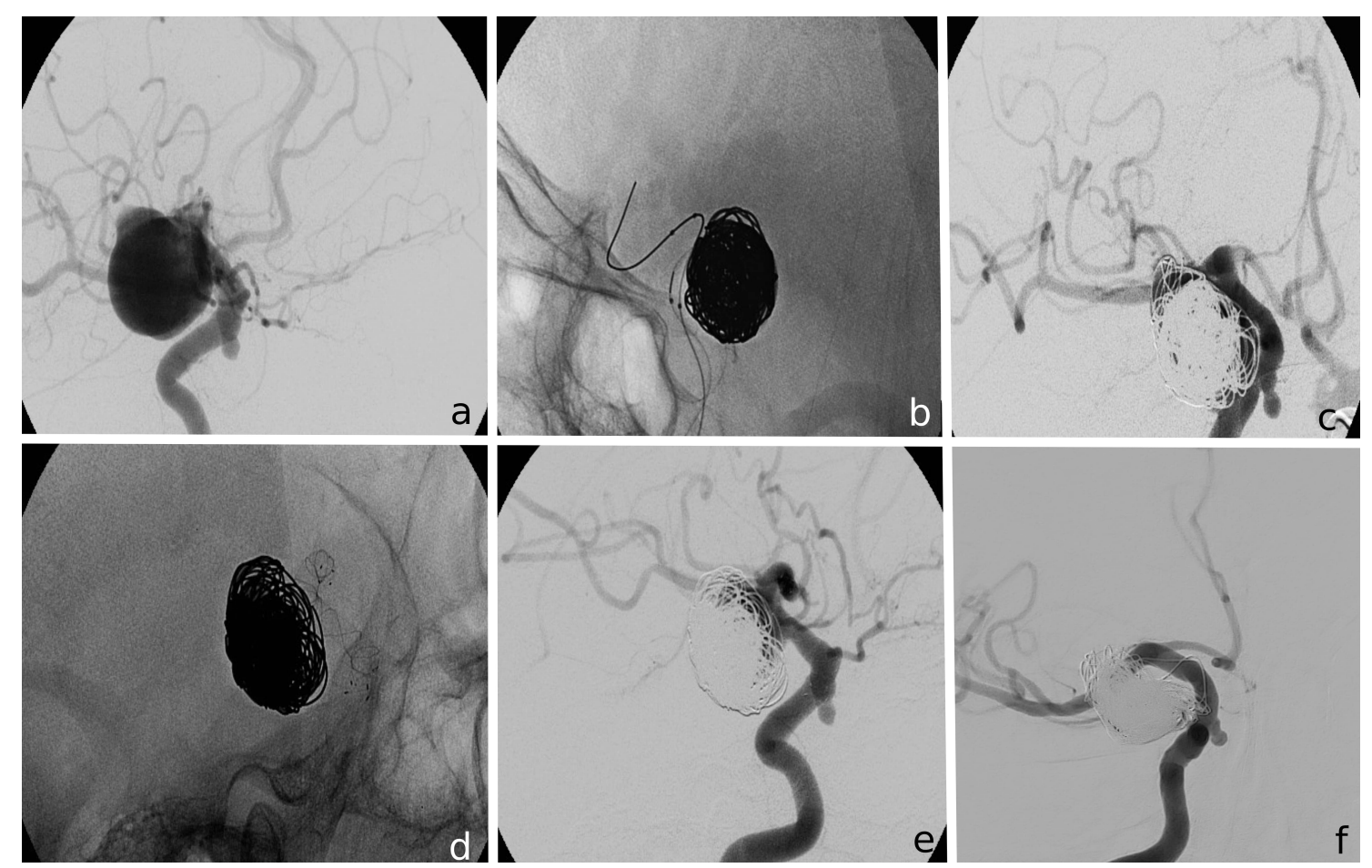

Fig. 2. DSA of stent assisted coiling of a ruptured PComA aneurysm. 70 years old woman with a history of subarachnoid hemorrhage and evidence of a giant PComA aneurysm at DSA (a). Endovascular treatment with balloon-assisted coiling (b) and complete aneurysm (c) occlusion. Because of the size of the aneurysm, 3 months later, a flow diverter was placed (d,e). Last follow-up (5 years) DSA shows the aneurysm's complete occlusion with no recanalization (f). 

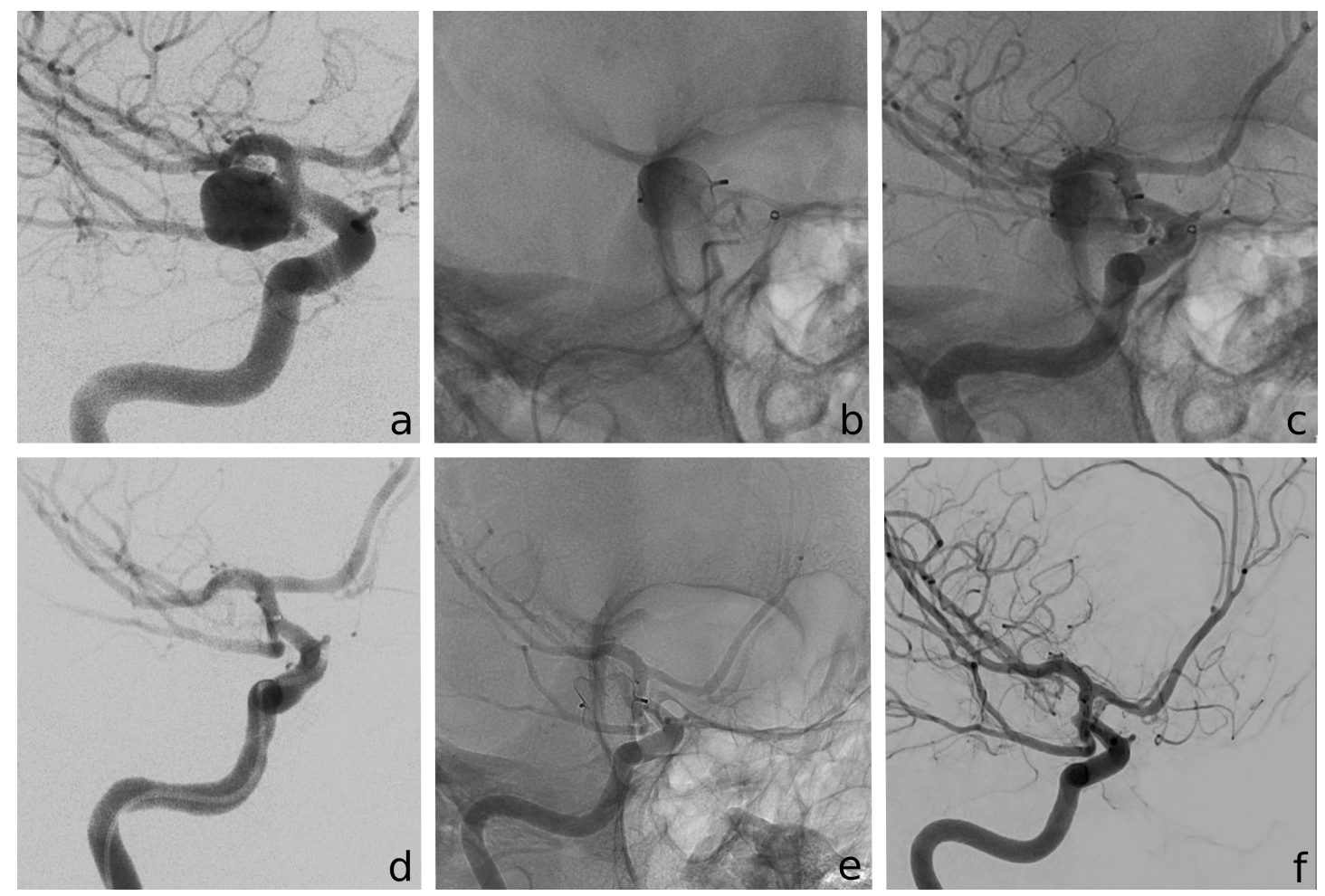

Fig. 3. DSA of intravascular device placement for the treatment of a ruptured PComA aneurysm. 45 years old woman with unruptured PComA aneurysms (a). Intravascular device deployment (b,c) with complete aneurysm (d) occlusion. Six-months follow-up DSA shows the aneurysm's complete occlusion (e,f).

canalization rates unrelated to PCA variation. Furthermore, fetal-type PCA failed to emerge as a risk factor for recanalization in multivariate analysis.

Coiling is the most common procedure which guarantees a good rate of immediate occlusion but a higher rate of longterm recanalization. It should be mainly proposed for ruptured aneurysms [38]. Flow-diversion is mainly effective in the long term, with no recanalization once that aneurysm occlusion is achieved. Long-term antiplatelet administration should be considered in general (not only cerebral) hemorrhagic risks.

\subsection{Limitations}

This is a retrospective study, flawed by all the inherent related bias. We did not include the clinical outcome of patients; as our primary aim was to investigate the radiological occlusion rate for the different types of treatments. While it is the largest study about endovascular Treatment of PComA aneurysms, the sample size could still reduce the power to detect differences between groups. Longer follow-up was available for a reduced number of patients. Results of intravascular devices are promising and should be better investigated with further specific prospective studies.

The multi-centric nature of this study could cause discrepancies in the type of deployed devices and drug administration protocols, with consequently outcome evaluation biases. Comorbidities would be very useful for a complete evaluation. Unfortunately, we only have data about hypertension, which was present in $45.3 \%$ of patients and was not shown to be a statistically significant factor $(p=0.6)$ for occlusion rate, recanalization, or complications.

\section{Conclusions}

Endovascular Treatment of PComA aneurysms is effective and presents a $15 \%$ rate of complications. No differences were detected in terms of occlusion rate in the fetal-type variant. Coiling guarantees a good rate of immediate occlusion and presents a higher recanalization rate, while flow diversion is effective, but in the longer term. Intravascular devices are promising. However, more cases are needed to draw more detailed conclusions. However, we should consider that our paper mainly focuses on the pure radiological technical aspect, lacking relevant clinical data.

\section{Abbreviations}

DSA, digital subtraction angiography; FD, flow-diverter; ICA, internal carotid artery; MRa, magnetic resonance angiography; PCA, posterior cerebral artery; PComA, posterior communicating artery; RR, Raymond-Roy classification; SAH, subarachnoid hemorrhage; TIA, Transient ischemic attacks; WEB, Woven EndoBridge.

\section{Author contributions}

ASc, MP, CLS and GT designed the research study. FS, PDB and ASa performed the research. AM, AT, VDE, VV, 
AP, IV, OR, GB, GI, LM, LR, AC, GP, collected patients data. ASc, CLS and GT analyzed the data. ASc and GT wrote the manuscript. All authors contributed to editorial changes in the manuscript. All authors read and approved the final manuscript.

\section{Ethics approval and consent to participate}

Patients gave written informed consent to use their medical and radiological data. The Institutional Review Board (AOSPFe EC) approved this study [EC 1254/M/2017].

\section{Acknowledgment}

We thank the anonymous reviewers for excellent criticism of the article.

\section{Funding}

This research received no external funding.

\section{Conflict of in terest}

The authors declare no conflict of interest.

\section{References}

[1] Ojemann RG. Internal carotid artery aneurysms. Surgical Management of Cerebrovascular Disease. 1988.

[2] Bisaria KK. Anomalies of the posterior communicating artery and their potential clinical significance. Journal of Neurosurgery. 1984; 60: $572-576$.

[3] Sturiale CL, Marchese E, Puca A, Olivi A, Albanese A. Surgical Treatment of Posterior Communicating Artery Aneurysms: Hints and Precautions for Young Cerebrovascular Surgeons. Journal of Neurological Surgery. 2019; 80: 205-212.

[4] Signorelli F, Pop R, Ganau M, Cebula H, Scibilia A, Gallinaro $\mathrm{P}$, et al. Endovascular versus surgical treatment for improvement of oculomotor nerve palsy caused by unruptured posterior communicating artery aneurysms. Journal of NeuroInterventional Surgery. 2020; 12: 964-967.

[5] Patel NJ, Morgan MK. ICA aneurysm surgically treated utilizing a choroidal to PCOM bypass and vein bypass. Neurosurgical Focus. 2015; 39: V14.

[6] Khan S, Smith T, Zomorodi A, McDonagh D, Agrawal A, Alexander M, et al. Effect of surgical clipping versus endovascular coiling on recovery from oculomotor nerve palsy in patients with posterior communicating artery aneurysms: a retrospective comparative study and meta-analysis. Asian Journal of Neurosurgery. 2013; 8: 117-124.

[7] Nakano Y, Saito T, Yamamoto J, Takahashi M, Akiba D, Kitagawa $\mathrm{T}$, et al. Surgical treatment for a ruptured true posterior communicating artery aneurysm arising on the fetal-type posterior communicating artery-two case reports and review of the literature. Journal of University of Occupational and Environmental Health. 2011; 33: 303-312.

[8] Aso K, Kashimura H, Akamatsu Y, Ogasawara Y, Oshida S. Cerebral Aneurysm Arising from Variant Posterior Communicating Artery Lying Lateral to Oculomotor Nerve. World Neurosurgery. 2019; 127: 478-480.

[9] Taweesomboonyat C, Tunthanathip T, Kaewborisutsakul A, Saeheng S, Oearsakul T, Riabroi K, et al. Outcome of Ruptured Posterior Communicating Artery Aneurysm Treatment Comparing between Clipping and Coiling Techniques. World Neurosurgery. 2019; 125: e183-e188

[10] Tomatis A, Trevisi G, Boido B, Perez R, Benech CA. Surgical Outcomes and their Correlation with Increasing Surgical Experience in a Series of 250 Ruptured or Unruptured Aneurysms Under- going Microsurgical Clipping. World Neurosurgery. 2019; 130: e542-e550.

[11] Della Pepa GM, Bianchi F, Scerrati A, Albanese A, Cotroneo E, Delitala A, et al. Secondary coiling after incomplete surgical clipping of cerebral aneurysms: a rescue strategy or a treatment option for complex cases? Institutional series and systematic review. Neurosurgical Review. 2019; 42: 337-350.

[12] Tsang ACO, Fung AMY, Tsang FCP, Leung GKK, Lee R, Lui WM. Failure of Flow Diverter Treatment of Intracranial Aneurysms Related to the Fetal-type Posterior Communicating Artery. Neurointervention. 2015; 10: 60-66.

[13] Kan P, Duckworth E, Puri A, Velat G, Wakhloo A. Treatment failure of fetal posterior communicating artery aneurysms with the pipeline embolization device. Journal of Neurointerventional Surgery. 2016; 8: 945-948.

[14] Kühn AL, Dabus G, Kan P, Wakhloo AK, Puri AS. Flow-diverter stents for endovascular management of non-fetal posterior communicating artery aneurysms-analysis on aneurysm occlusion, vessel patency, and patient outcome. Interventional Neuroradiology. 2018; 24: 363-374.

[15] Wallace AN, Kayan Y, Austin MJ, Delgado AlmandozJE, Kamran $\mathrm{M}$, Cross DT, et al. Pipeline embolization of posterior communicating artery aneurysms associated with a fetal origin posterior cerebral artery. Clinical Neurology and Neurosurgery. 2017; 160: 83-87.

[16] Brinjikji W, Lanzino G, Cloft HJ, Kallmes DF. Patency of the posterior communicating artery after flow diversion treatment of internal carotid artery aneurysms. Clinical Neurology and Neurosurgery. 2014; 120: 84-88.

[17] Rustemi O, Raneri F, Scerrati A. Letter by Rustemi et al Regarding Article, "Flow Diverters for Intracranial Aneurysms: the DIVERSION National Prospective Cohort Study". Stroke. 2020; 51: e35.

[18] Choi HH, Cho YD, Yoo DH, Lee HS, Kim S, Jang D, et al. Impact of fetal-type posterior cerebral artery on recanalization of posterior communicating artery aneurysms after coil embolization: matched-pair case-control study. Journal of NeuroInterventional Surgery. 2020; 12: 783-787.

[19] Roy D, Milot G, Raymond J. Endovascular treatment of unruptured aneurysms. Stroke. 2001; 32: 1998-2004.

[20] Endo H, Sato K, Kondo R, Matsumoto Y, Takahashi A, Tominaga $\mathrm{T}$. Tuberothalamic artery infarctions following coil embolization of ruptured posterior communicating artery aneurysms with posterior communicating artery sacrifice. American Journal of Neuroradiology. 2012; 33: 500-506.

[21] Lv X, Li Y, Yang X, Jiang C, Wu Z. Potential proneness of fetaltype posterior cerebral artery to vascular insufficiency in parent vessel occlusion of distal posterior cerebral artery aneurysms. Journal of Neurosurgery. 2012; 117: 284-287.

[22] Cho YD, Jung SC, Kim CH, Ahn JH, Kang H, Kim JE, et al. Posterior Communicating Artery Compromise in Coil Embolization of Posterior Communicating Artery Aneurysms. Clinical Neuroradiology. 2015; 25: 275-279.

[23] Raymond J, Guilbert F, Weill A, Georganos SA, Juravsky L, Lambert $\mathrm{A}$, et al. Long-term angiographic recurrences after selective endovascular treatment of aneurysms with detachable coils. Stroke. 2003; 34: 1398-1403.

[24] Campi A, Ramzi N, Molyneux AJ, Summers PE, Kerr RSC, Sneade $\mathrm{M}$, et al. Retreatment of ruptured cerebral aneurysms in patients randomized by coiling or clipping in the International Subarachnoid Aneurysm Trial (ISAT). Stroke. 2007; 38: 1538-1544.

[25] el-Chalouhi N, Jabbour PM, Tjoumakaris SI, Starke RM, Dumont AS, Liu $\mathrm{H}$, et al. Treatment of large and giant intracranial aneurysms: cost comparison of flow diversion and traditional embolization strategies. World Neurosurgery. 2014; 82: 696-701.

[26] Aikawa H, Kazekawa K, Nagata S, Onizuka M, Iko M, Tsutsumi $\mathrm{M}$, et al. Rebleeding after endovascular embolization of ruptured cerebral aneurysms. Neurologia Medico-Chirurgica. 2007; 47: 439-437.

[27] Zada G, Breault J, Liu CY, Khalessi AA, Larsen DW, Teitelbaum GP, et al. Internal carotid artery aneurysms occurring at the origin 
of fetal variant posterior cerebral arteries: surgical and endovascular experience. Neurosurgery. 2008; 63: ONS55-ONS62.

[28] Darkwah Oppong M, Gembruch O, Pierscianek D, Köhrmann M, Kleinschnitz C, Deuschl C, et al. Post-treatment Antiplatelet Therapy Reduces Risk for Delayed Cerebral Ischemia due to Aneurysmal Subarachnoid Hemorrhage. Neurosurgery. 2019; 85: 827833.

[29] Nagahama Y, Allan L, Nakagawa D, Zanaty M, Starke RM, Chalouhi $\mathrm{N}$, et al. Dual antiplatelet therapy in aneurysmal subarachnoid hemorrhage: association with reduced risk of clinical vasospasm and delayed cerebral ischemia. Journal of Neurosurgery. 2018; 129: 702-710.

[30] König I, Maurer C, Berlis A, Maus V, Weber W, Fischer S. Treatment of Ruptured and Unruptured Intracranial Aneurysms with WEB 17 Versus WEB 21 Systems: Comparison of Indications and Early Angiographic Outcomes. Clinical Neuroradiology. 2020; 31: 691-697.

[31] Pierot L, Biondi A, Narata A-, Mihalea C, Januel AC, Metaxas G, et al. Should indications for WEB aneurysm treatment be enlarged? Report of a series of 20 patients with aneurysms in "atypical" locations for WEB treatment. Journal of Neuroradiology. 2017; 44: 203-209.

[32] Bhogal P, Brouwer PA, Yeo L, Svensson M, Söderman M. The Medina Embolic Device: Karolinska experience. Interventional Neuroradiology. 2018; 24: 4-13.

[33] Cagnazzo F, Ahmed R, Dargazanli C, Lefevre P-, Gascou G, Derraz I, et al. Treatment of Wide-Neck Intracranial Aneurysms with the Woven EndoBridge Device Associated with Stenting: a Single-Center Experience. American Journal of Neuroradiology. 2019; 40: 820-826.

[34] Scerrati A, Visani J, Flacco ME, Ricciardi L, Trungu S, Raco A, et al. Endovascular Treatment of Ruptured Intracranial Blister Aneurysms: a Systematic Review and Meta-analysis. American Journal of Neuroradiology. 2020; 42: 538-545.

[35] Roy AK, Howard BM, Haussen DC, Osbun JW, Halani SH, Skukalek SL, et al. Reduced Efficacy of the Pipeline Embolization Device in the Treatment of Posterior Communicating Region Aneurysms with Fetal Posterior Cerebral Artery Configuration. Neurosurgery. 2018; 82: 695-700.

[36] Zanaty M, Chalouhi N, Starke RM, Jabbour P, Ryken KO, Bulsara KR, et al. Failure of the Pipeline Embolization Device in Posterior Communicating Artery Aneurysms Associated with a Fetal Posterior Cerebral Artery. Case Reports in Vascular Medicine. 2016; 2016: 4691275

[37] Enriquez-Marulanda A, Salem MM, Ascanio LC, Maragkos GA, Gupta R, Moore JM, et al. No differences in effectiveness and safety between pipeline embolization device and stent-assisted coiling for the treatment of communicating segment internal carotid artery aneurysms. The Neuroradiology Journal. 2019; 32: 344352.

[38] Bäcker HC, Shoap S, Vajda J, Nyáry I. Anterior communicating artery aneurysm rupture and functional outcome in short-term: clipping versus coiling. Journal of Integrative Neuroscience. 2020; 19: 349-354. 